\title{
Study on Application of Location Algorithm Base Multidimensional Spatial Information in the Situation Analysis of Natural Ecology*
}

\author{
Jumei $\mathrm{Ai}^{1}$ and Shuhua $\mathrm{Mao}^{2}$ \\ ${ }^{1}$ Key Laboratory of Radioactive Geology and Exploration Technology Fundamental Science for \\ National Defense, East China Institute of Technology, Fuzhou City, Jiangxi, 344000, China \\ jmai@ecit.cn \\ ${ }^{2}$ School of Information Engineering, East China Institute of Technology, Jiangxi, China
}

\begin{abstract}
In this paper, the algorithm was designed of quad-tree spatial search based on spatial information multi-dimensional features, the traditional quad-tree algorithm has been improved to a specific spatial attribute value binding in the quad-tree leaf nodes, a large number with a specific operation irrelevant attributes are excluded, to the effect of finding targets. This algorithm is applied in the natural ecology protection system, it can locate the region which pollutes quickly in the map, provides the scientific basis for the government in carrying on the ecological environment and using the resources environment sustainable.
\end{abstract}

Keywords: Spatial search, Quad-tree, Ecological equilibrium, Attribute binding.

\section{Introduction}

As a result of the urbanized advancement aggravating, the ecology uses the quantity large scale reduction moreover the quality also obviously to reduce, not only destroyed the ecosystem balance, also causes the human survival environment the worsening, thus cay, the society, the ecology development not to be uncoordinated. Therefore, in our country economic society fast development, the environmental protection and the ecology construction idea strengthened, the ecology construction unceasingly today which needed to strengthen continually to the national territory resource management, the ecological environment protection question already in relief comes out. The ecological environment information has the multi-domains, the multi-professions, the multi-varieties and so on the complex characteristic.

At present, has some specialized software to carry on processing to these information, if the GIS software aims at the geography information, because the geography information has the complex spatial attribute, pays attention to the hot spot to the spatial geography information fast search into people, this article proposed one kind based on Quad-tree search algorithm, can remove effectively the non-correlated information, locates fast to is interested the attribute special characteristic[1], [2].

* This work is supported by Project of Scientific and Technical Supporting Programs of Jiangxi Province (2007GCJ173). 


\section{The Design of Quad-Tree Location Algorithm}

Quadtree is a suitable algorithm in two-dimensional picture localization pixel. Because in the two-dimensional space, the plane pixel may be divided into four parts repeatedly, the depth of three is determined by the figure, the memory and the complexity of the figure. As an auxiliary multi-dimensional data structure of spatial index, quadtree lies between spatial operation algorithm. Many spatial objects which are independent of special spatial are excluded through its screening, and it enables the spatial operation to access operation object fast, thus enhances the efficiency of spatial operation[4].

Taking the ID $=0$ of layer as element as example:

A. Readout the element numbers and its border which layer contains by shapefile, the rectangule of figure 1 is the tree root node;

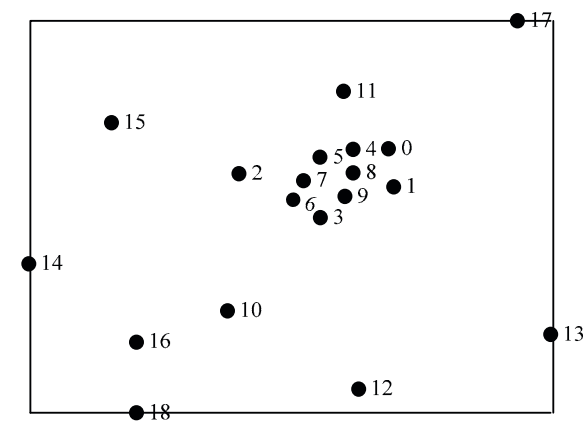

Fig. 1. The quadrant division when the point's depth is 1

B. Determine the max depth of tree according to the elements' total number.

C. Readout the border and ID number of every element circularly, build quadtree; using the rectangular overlap skill to avoid excessively too much elements distributing in the boundary in MapServer; recursive by the above algorithm until every element is assigned into the quadtree which is generated at last. Figure 2 is the quadrant division when the element point depth is 3 .

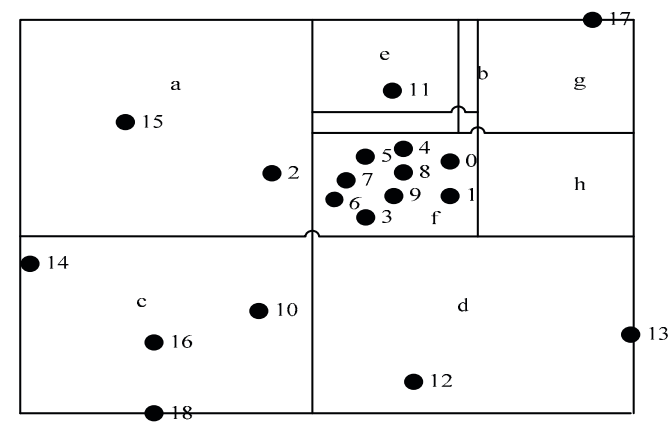

Fig. 2. The quadrant division when the point's depth is 3 


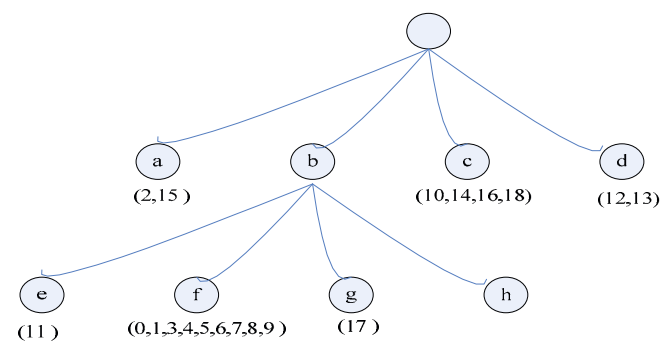

Fig. 3. Generating quadtree

\section{Application Example}

Using Quadtree search algorithm, may in the comprehensive analysis ecological environment each kind of data, help the establishment environmental effect appraisal model. Because the GIS information has the multi-dimensional structure, may carry on the different environmental effect calculates and superimposes. In the region environment quality present situation appraisal work, may environment essential factor and so on geography information and atmosphere, soil, water, noise monitor data unifies in together, uses the GIS software the spatial analysis module, carries on objectively, the comprehensive appraisal to the entire region environment quality present situation, reflected in the region the degree as well as the spatial distribution situation which pollutes. If through the superimposition analysis, uses Quadtree search algorithm to be possible to locate in fast this region the air pollution butut, the noise distribution map; Through the buffer analysis, may demonstrate the source of pollution influence scope and so on.

\section{The Efficiency Analysis of Mobile Map Search Algorithm}

This algorithm used in the Mobile Map Search system base on MMS. The recall ratio and precision ratio efficiency are better. A new assessment index named comprehensive assessment rate $\mathrm{F}$ (the factor of satisfaction) is obtained by considering the recall ratio and precision ratio comprehensively. The formula for calculation [3] is as follows:

$$
\mathrm{F}=\frac{\text { precision } \times \text { recall } \times 2}{\text { precision }+ \text { recall }}
$$

In this experiment, www.google.cn and www.baidu.com are setup as under-layered search-engine for meta-search. 2319 documents (1560 documents from Google, 759 documents from Baidu and delete the same document) related by the public infrastructure construction and development of Chengdu city are obtained from the Internet. These documents will be divided into five categories artificially including public transportation information, railway information, flight information, tourist sites and customary snacks. The vector space dimension of different categories of documents is obtained by characteristic word segmentation processing [3]. 
The dataset used in the experiment as follows: public transportation information: 235, railway information: 450, flight information: 209, tourist sites: 602, customary snacks: 823.

Two comparison experiments are done to the 2319 documents:

(1) Take advantage of traditional search-engine stochastic querying the dataset, statistic the recall ratio and precision ratio, and then calculate the factor of satisfaction $(F)$.

(2) Use the query vector of the first experiment and the mobile map search algorithm that the paper proposed, statistic the recall ratio and precision ratio, calculate the factor of satisfaction as the return result.

The factor of satisfaction of traditional search-engine is about 46 percent, and the result of the factor of satisfaction which the Mobile Map Search Algorithm which this paper proposed returns is about 53.27 percent, and the precision ratio heightens. Therefore, the efficiency of Mobile Map Search Algorithm based on Quad-tree can be fit with the mobile search characteristic of shortcut and precision.

\section{Conclusion}

Along with information technology development, geographic information system technology, from two-dimensional to three dimensional development, from the static state data processing to the dynamic development, has the succession data-handling capacity, how uses the existing information infrastructure condition and the geography space data resources, the union numeral city, the electronic government affairs and so on serves well for the user, has become technological development and so on current geographic information system key contents. Based on Quadtree search algorithm to lay the foundation for the next generation GIS technology. In this paper, the quad-tree algorithm has been improved to a specific spatial attribute value binding in the quad-tree leaf nodes, a large number with a specific operation irrelevant attributes are excluded, to the effect of finding targets. This algorithm is applied in the natural ecology protection system, it can locate the region which pollutes quickly in the map, provides the scientific basis for the government in carrying on the ecological environment and using the resources environment sustainable.

\section{References}

1. iResearch. China Wireless Value-Added Service Market Report

2. Zhang, L., Guo, B., Zhang, C.-w., Shen, Y.: Mobile Search Secondary Ordering Algorithm Based on Short Message. Computer Engineering, 43-45 (2008)

3. Crane, D., Pascarello, E., James, D.: ajaxcn.org, AjaxPractice. Beijing: People's posts and telecommunications publishing house (2006)

4. Zhao, B., Bian, F.-1.: Dynamic Quadtree Spatial Index Algorithm for Mobile GIS. Computer Engineering 3(1), 86-87 (2007) 